\title{
Rogue Wave Observation in a Water Wave Tank
}

\author{
A. Chabchoub, ${ }^{1, *}$ N. P. Hoffmann, ${ }^{1}$ and N. Akhmediev ${ }^{2}$ \\ ${ }^{1}$ Mechanics and Ocean Engineering, Hamburg University of Technology, Eißendorfer Straße 42, 21073 Hamburg, Germany \\ ${ }^{2}$ Optical Sciences Group, Research School of Physics and Engineering, The Australian National University, \\ Canberra ACT 0200, Australia
}

(Received 28 February 2011; published 16 May 2011)

\begin{abstract}
The conventional definition of rogue waves in the ocean is that their heights, from crest to trough, are more than about twice the significant wave height, which is the average wave height of the largest onethird of nearby waves. When modeling deep water waves using the nonlinear Schrödinger equation, the most likely candidate satisfying this criterion is the so-called Peregrine solution. It is localized in both space and time, thus describing a unique wave event. Until now, experiments specifically designed for observation of breather states in the evolution of deep water waves have never been made in this double limit. In the present work, we present the first experimental results with observations of the Peregrine soliton in a water wave tank.
\end{abstract}

DOI: 10.1103/PhysRevLett.106.204502

PACS numbers: 47.35.Fg, 92.10.Hm

Today, there is widespread consensus on the existence of rogue waves in the ocean [1-3]. A number of mechanisms have been proposed to explain their unexpected emergence. One of the essential elements in many of these explanations is the idea that rogue waves could be related to breather-type solutions of the underlying evolution equations [4-6]. Such solutions could, in principle, describe a large wave that appears from nowhere and disappears without a trace [7], a behavior that has been reported for many known rogue wave events. One of the most direct approaches to modeling the evolution of gravity water waves is the use of the nonlinear Schrödinger equation (NLS) $[4,8,9]$, which is known to be good for capturing weakly nonlinear evolution of narrow-band processes. It is now already more than 30 years since the first breather-type solution of the NLS was found by Ma [10]. This solitonlike solution breathes temporally but is spatially localized. Taking the temporal period of this solution to infinity, Peregrine, in 1983, gave a solution localized in both space and time [11]. Akhmediev has found a family of solutions $[12,13]$ qualitatively different from Ma breathers. These solutions breathe spatially but are localized in time. Simply speaking, Akhmediev breathers are exact solutions of the NLS that start from modulation instability of a plane wave [13] (also known as a Benjamin-Feir [14-17] or Bespalov-Talanov instability) and return to a plane wave at the end of the evolution. Remarkably, the Peregrine solution is also a limiting case of an Akhmediev breather when the spatial period is taken to be infinite.

Surprisingly, to date, there does not seem to be any published study focusing on direct experimental observation of any of the above described breather solutions in a physical wave tank. Although a number of experimental investigations have focused on the Benjamin-Feir instability and focusing-defocusing issues (see, e.g., [18] for the most recent work), none of these studies seems to have focused on generating and studying breather solutions with complete growth-decay cycles. The question of how well breather solutions of the NLS would correspond to breather solutions of physical surface gravity waves has thus generated discussions. But sound comparison between experimental data and the NLS-based prediction is still lacking, although such a comparison is generally considered to be an important issue, since many authors have realized that breather solutions might be closely related to rogue waves in the ocean [4-7].

In the present study (i) we observe a Peregrine-type breather solution experimentally in a water wave tank, and (ii) we make a direct comparison of measured results with the predictions of the analytical solutions of the NLS. These comparisons bring us to the conclusion that our experiment is the first observation of an isolated rogue wave in a water wave tank.

Deep water waves can be described by the NLS, first derived by Zakharov [16]:

$$
i\left(\frac{\partial a}{\partial t}+c_{g} \frac{\partial a}{\partial x}\right)-\frac{\omega_{0}}{8 k_{0}^{2}} \frac{\partial^{2} a}{\partial x^{2}}-\frac{\omega_{0} k_{0}^{2}}{2}|a|^{2} a=0,
$$

where $t$ and $x$ are time and longitudinal coordinates, while $k_{0}$ and $\omega_{0}=\omega\left(k_{0}\right)$ denote the wave number and the frequency of the carrier wave, respectively. $\omega_{0}$ and $k_{0}$ are linked by the dispersion relation of the linear deep water wave theory, $\omega_{0}=\sqrt{g k_{0}}$, where $g$ denotes the gravitational acceleration. Accordingly, the group velocity is $c_{g}:=\left.\frac{d \omega}{d k}\right|_{k=k_{0}}=\frac{\omega_{0}}{2 k_{0}}$. The surface elevation $\eta(x, t)$ of the sea water is then given by $\eta(x, t)=$ $\operatorname{Re}\left\{a(x, t) \exp \left[i\left(k_{0} x-\omega_{0} t\right)\right]\right\}$.

A dimensionless form of the NLS [19],

$$
i q_{T}+q_{X X}+2|q|^{2} q=0
$$

is obtained from (1) by using the rescaled variables: 


$$
\begin{gathered}
T=-\frac{\omega_{0}}{8 k_{0}^{2}} t, \quad X=x-c_{g} t=x-\frac{\omega_{0}}{2 k_{0}} t, \\
q=\sqrt{2} k_{0}^{2} a .
\end{gathered}
$$

Here, $X$ is the coordinate in the frame moving with the wave group velocity, and $T$ is the time. Note that for $a \in \mathbb{R}$ the scaling transformation $X \rightarrow a X, T \rightarrow a^{2} T, q \rightarrow \frac{q}{a}$ leaves the NLS (2) invariant. This implies that if $q(X, T)$ is a solution of (2), then so is $a q\left(a X, a^{2} T\right)$. Initial value problems involving the NLS can be solved by using the inverse scattering transform [19]. On the other hand, there are a number of exact solutions to the NLS that describe various simpler physical situations. In particular, a number of stationary solutions and nonstationary breather-type solutions are known. Ma [10] found a breather solution of (2), which is periodic in time and tends to the plane wave solution as $X \rightarrow \pm \infty$ :

$$
q_{M}(X, T)=\frac{\cos (\Omega T-2 i \varphi)-\cosh (\varphi) \cosh (p X)}{\cos (\Omega T)-\cosh (\varphi) \cosh (p X)} e^{(2 i T)},
$$

$\Omega=2 \sinh (2 \varphi), p=2 \sinh (\varphi)$ and $\varphi \in \mathbb{R}$. Simply speaking, Eq. (3) is a soliton on a background. A similar solution for the NLS with an additional term has been found by Kuznetsov [20]. Akhmediev [12,13] proved the existence of a family of space-periodic solutions of (2) which approaches the plane wave as $T \rightarrow \pm \infty$ :

$$
q_{A}(X, T)=\frac{\cosh (\Omega T-2 i \varphi)-\cos (\varphi) \cos (p X)}{\cosh (\Omega T)-\cos (\varphi) \cos (p X)} e^{(2 i T)},
$$

where $\Omega=2 \sin (2 \varphi), p=2 \sin (\varphi)$, and $\varphi \in \mathbb{R}$. It is easy to show that Eq. (4) is an exact solution of the NLS that starts with modulation instability [13].

The Peregrine breather [11] can be understood as a limiting case of the solutions (3) and (4) when $\varphi \rightarrow 0$, i.e., when the period of either solution becomes infinite:

$$
\begin{aligned}
q_{P}(X, T) & =\lim _{\varphi \rightarrow 0} q_{A}(X, T)=\lim _{\varphi \rightarrow 0} q_{M}(X, T) \\
& =\left(1-\frac{4(1+4 i T)}{1+4 X^{2}+16 T^{2}}\right) e^{(2 i T)} .
\end{aligned}
$$

A remarkable feature of this solution is that it is localized in both space and time, and, as such, it describes unique wave events. Namely, it breathes only once and describes a single wave that appears from nowhere and disappears without a trace. Figure 1 shows this solution, demonstrating clearly its spatial and temporal localization. Since the Peregrine breather is the simplest solution of the NLS which is localized in both space and time (the whole hierarchy is much more complicated [7]), it has been conjectured to sort of form a prototype for rogue waves, in general, and also in the ocean [5,6]. However, there has been no observational or experimental confirmation of this. Observations of rogue waves in the ocean are rare and therefore typically incomplete, and experiments in the

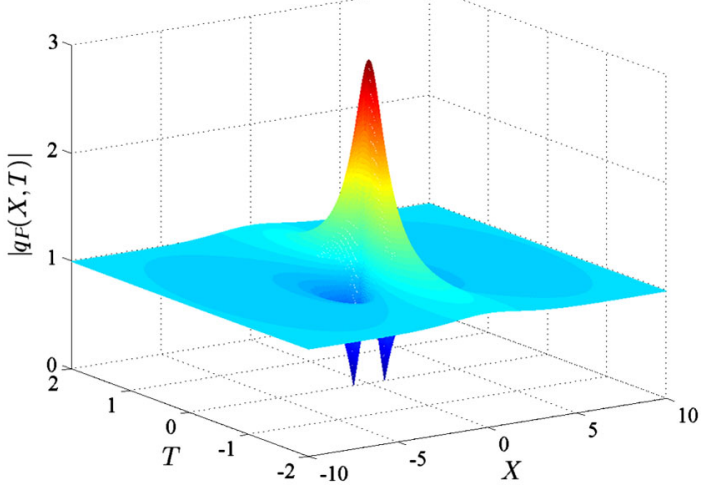

FIG. 1 (color online). Peregrine breather solution (5). The maximum amplitude, which occurs at $X=0, T=0$, is a factor of 3 higher than the background carrier wave. Note also the sharp drops in the amplitude (troughs) on either side of the maximum.

open ocean are dangerous and virtually impossible. Experiments in water wave tanks, until now, do not seem to have been conducted, either. Recent experiments in fiber optics [21] are impressive but cannot be automatically assumed to describe water waves.

We have performed the experiments in a $15 \mathrm{~m} \times$ $1.6 \mathrm{~m} \times 1.5 \mathrm{~m}$ water wave tank with $1 \mathrm{~m}$ water depth. The tank is depicted schematically in Fig. 2. A singleflap paddle activated by a hydraulic cylinder is located at one end of the tank. To avoid wave reflections from the opposite end of the tank, there is a wave-absorbing beach there. The surface elevation of water at a given point is measured by a capacitance wave gauge with a sensitivity of $1.06 \mathrm{~V} / \mathrm{cm}$ and a sampling frequency of $500 \mathrm{~Hz}$.

In order to describe experimental results, the Peregrine breather solution has to be written in dimensional units. With that aim, we apply the transformation [4]

$$
a X \rightarrow \sqrt{2} k_{0}^{2} a_{0}\left(x-c_{g} t\right), \quad a^{2} T \rightarrow-\frac{k_{0}^{2} a_{0}^{2} \omega_{0}}{4} t .
$$

Thus, the solution becomes

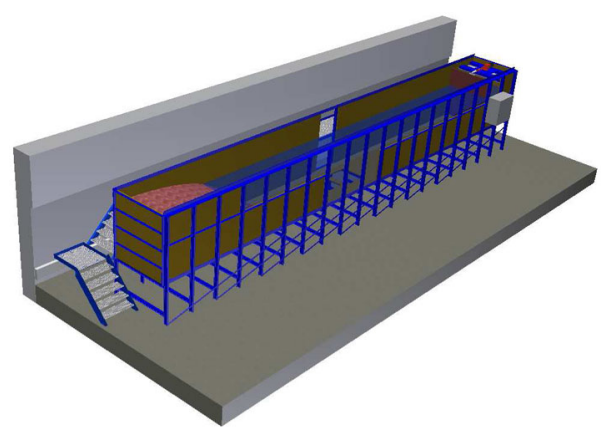

FIG. 2 (color online). Schematic illustration of the water wave tank. 


$$
\begin{aligned}
q_{P}(x, t)= & a_{0} \exp \left(-\frac{i k_{0}^{2} a_{0}^{2} \omega_{0}}{2} t\right) \\
& \times\left(1-\frac{4\left(1-i k_{0}^{2} a_{0}^{2} \omega_{0} t\right)}{1+\left[2 \sqrt{2} k_{0}^{2} a_{0}\left(x-c_{g} t\right)\right]^{2}+k_{0}^{4} a_{0}^{4} \omega_{0}^{2} t^{2}}\right) .
\end{aligned}
$$

Before starting the first experiments, we expected that the main experimental difficulty would be in determining the flap kinematics. Ideally, the water motion created by the flap should correspond to the initial conditions given by the Peregrine breather solution at the starting side of the tank. As a first step, the flap displacement was chosen to be proportional to the surface height of the breather solution to be generated. Surprisingly, the resulting wave dynamics, described below, was so close to the analytical predictions that we concluded that the excitation arrangement turned out to be perfectly sufficient for the present study. All experiments described below were therefore performed with a flap motion which was directly proportional to the surface elevation given by (7) at the side where we define the initial conditions.

An important point is that the Peregrine soliton is located on a background wave which provides energy for its growth. The choice for the parameters of this wave is dictated by the size of the tank. In all tests, the dimensional far-field amplitude of the background was selected to be $a_{0}=0.01 \mathrm{~m}$. The wavelength of the carrier has been set to $\lambda=0.54 \mathrm{~m}$, corresponding to a wave number of about $k_{0}=11.63 \mathrm{~m}^{-1}$ and an angular frequency of about $\omega_{0}=10.7 \mathrm{~s}^{-1}$. These values have been chosen in order to ensure that the wavelength is large enough to ignore effects of surface tension but still small enough to have sufficient tank length to develop the wave evolution described by Eq. (7). The wavelength also has to be small enough for the whole arrangement to be sufficiently close to the deep water limit.

Once the dimensional Peregrine breather solution is defined and the background wave parameters are specified, the theoretical spatiotemporal surface-height distribution in dimensional units is given by

$$
\eta(x, t)=\operatorname{Re}\left\{q_{P}(x, t) \exp \left[i\left(k_{0} x-\omega_{0} t\right)\right]\right\} .
$$

This formula is used both to determine the initial conditions for the wave maker's paddle motion and for comparing measured surface-height time series with theoretical predictions. The position where the rogue wave develops its maximum amplitude depends on the initial conditions at the wave maker. In order to demonstrate the evolution of a nearly periodic Stokes wave towards the most extreme wave state, we have initially chosen the position of the maximum as far in the direction of wave propagation in the channel as feasible. Figure 3 shows the results.

We arranged a carrier wave yielding the maximum breather amplitude about $9 \mathrm{~m}$ along the tank. Water surface-height data have been collected at ten positions,

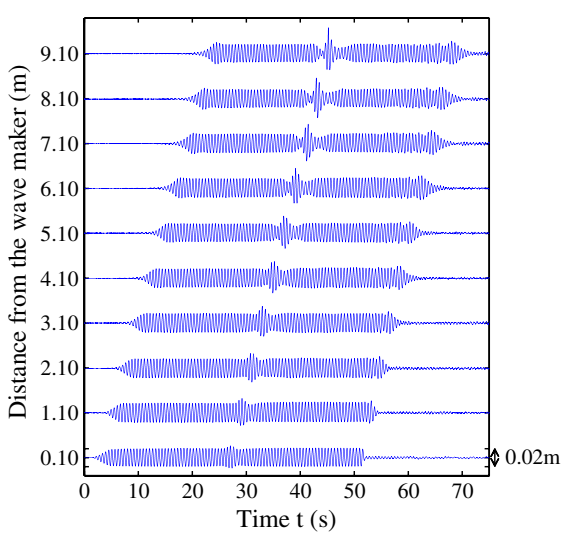

FIG. 3 (color online). Temporal evolution of the water surface height at various distances from the wave maker.

with equal separations of $1 \mathrm{~m}$, along the direction of wave propagation. The measurements show that the wave is essentially sinusoidal when close to the flap. This can be seen clearly from the wave profile measured at $10 \mathrm{~cm}$ next to the mean flap position. The flap motion produces a periodic wave with about $1 \mathrm{~cm}$ amplitude, with a brief increase of modulation above that level to about $1.4 \mathrm{~cm}$ in the middle of the packet. Measurements at further distances from the wave maker show, surprisingly well, the process of amplitude growth of this perturbation which remains strongly localized and moves along with group velocity.

The top curve in Fig. 3 is measured at the position where we expect the growing rogue wave to have its maximum amplitude. A closer look at this last time series at $x=9.1 \mathrm{~m}$ also shows that the almost perfect periodicity of the background wave packet is slightly disturbed. Namely, reflected waves from the absorbing beach at the end of the tank can already be seen at the end of the wave packet. However, they arrive at the position where the measurements are taken only after the breather has already passed. Interference with the waves reflected from the absorbing beach makes it difficult in the present wave

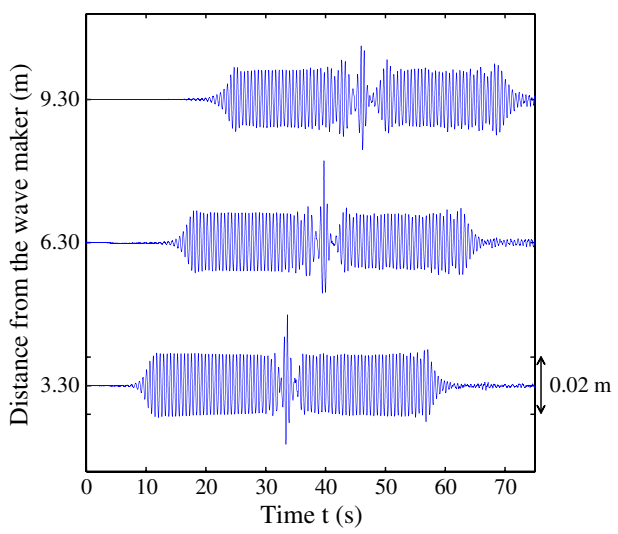

FIG. 4 (color online). Peregrine soliton measured at the growing, as well as decaying, stages of its evolution. 


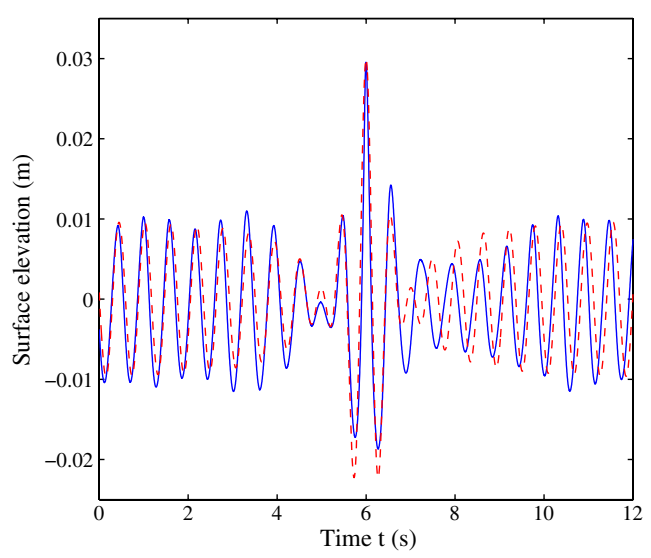

FIG. 5 (color online). Comparison of measured surface height at the position of maximum rogue wave amplitude (solid line) with the theoretical Peregrine solution (dashed line) evaluated at $X=0$.

tank to visualize the decay of the localized solution with the same quality as its growth. To overcome this limitation of our apparatus, we programed the motion of the wave maker to create initial conditions that bring the extreme state closer to the starting point of the wave propagation. The results of these measurements are shown in Fig. 4. Here we have chosen the position of the maximum amplitude to be about $6.3 \mathrm{~m}$ from the wave maker. This choice gives us a chance to watch the decaying stage of the rogue wave. Indeed, the measurements taken at $9.3 \mathrm{~m}$ further down the tank show that the amplitude of the perturbation decreases.

Our experimental observation of the localization of the wave energy in both space and time is a confirmation of the existence of Peregrine solitons on the water surface, thus confirming the existence of rogue waves "that appear from nowhere." The theoretical prediction from the Peregrine solution suggests that the carrier wave surface elevation should be amplified by a factor of 3. The measurements come very close to this value. Figure 5 represents a surfaceheight measurement at a position close to the presumed maximum envelope amplitude. While the carrier wave has an amplitude of about one centimeter, the surface height of the "maximum wave" of the breather almost exactly reaches a value of three centimeters.

By comparing the measured time series (solid line) with the curve predicted by the Peregrine solution (dashed line), the agreement can be considered to be reasonable. First, the periodic wave state reveals a deep trough right next to the breather's maximum wave on either side of the wave crest. The wave height from crest to trough here is almost 2.5 times larger than the average wave height around the perturbation. This measure by itself satisfies the definition of a rogue wave, for which the wave height should exceed at least 2.2 times the significant wave height [22]. Second, the return from the state of the extreme wave back to the state of a periodic wave in the experiment is almost symmetric in time, just as in theory. We attribute this correspondence to the narrowness of the frequency spectrum in this particular problem. The spectrum of the Peregrine soliton consists of the delta function defined by the background monochromatic wave plus a small deviation from it in the form of a triangular spectrum. We confirmed, experimentally, that the whole spectrum remains narrow as required for the NLS to be valid. Nevertheless, some asymmetry in the surface elevation can be noticed and will be the subject of future studies taking into account extended modeling approaches.

A. C. and N. P. H. thank M.-A. Pick for support with the experimental setup and C. Weiß and O. Mahrenholtz for discussions concerning the experiments. N. A. and N.P. H. acknowledge the support of the VolkswagenStiftung. N. A. acknowledges partial support of the Australian Research Council (Discovery Project No. DP110102068). N. A. is supported by a Alexander von Humboldt Award.

*amin.chabchoub@tuhh.de

[1] C. Kharif, E. Pelinovsky, and A. Slunyaev, Rogue Waves in the Ocean (Springer, New York, 2009).

[2] C. Garrett and J. Gemmrich, Phys. Today 62, No. 16, 57 (2009).

[3] P. Müller, C. Garrett, and A. Osborne, Oceanography 18, 66 (2005).

[4] A. Osborne, Nonlinear Ocean Waves and the Inverse Scattering Transform (Elsevier, New York, 2010).

[5] K. B. Dysthe and K. Trulsen, Phys. Scr. T82, 48 (1999).

[6] V. I. Shrira and V. V. Geogjaev, J. Eng. Math. 67, 11 (2009).

[7] N. Akhmediev, A. Ankiewicz, and M. Taki, Phys. Lett. A 373, 675 (2009).

[8] H. C. Yuen and B. M. Lake, Phys. Fluids 18, 956 (1975).

[9] B. M. Lake et al., J. Fluid Mech. 83, 49 (1977).

[10] Y.C. Ma, Stud. Appl. Math. 60, 43 (1979).

[11] D. H. Peregrine, J. Aust. Math. Soc. Series B, Appl. Math. 25, 16 (1983).

[12] N. Akhmediev, V. M. Eleonskii, and N. Kulagin, Zh. Eksp. Teor. Fiz. 98, 1542 (1985).

[13] N. Akhmediev and V.I. Korneev, Theor. Math. Phys. 69, 1089 (1986).

[14] M. J. Lighthill, J. Inst. Math. Appl. 1, 269 (1965).

[15] T.B. Benjamin and J.E. Feir, J. Fluid Mech. 27, 417 (1967).

[16] V.E. Zakharov, J. Appl. Mech. Tech. Phys. 9, 190 (1968).

[17] H.C. Yuen and B. M. Lake, Adv. Appl. Mech. 22, 67 (1982).

[18] N. Karjanto and E. Van Groesen, J. Hydro-environment Res. 3, 186 (2010).

[19] V.E. Zakharov and A. B. Shabat, Sov. Phys. JETP 34, 62 (1972).

[20] E. Kuznetsov, Dokl. Akad. Nauk SSSR 235, 575 (1977).

[21] B. Kibler et al., Nature Phys. 6, 790 (2010).

[22] Rogue waves-Towards a unifying concept?: Discussions and debates [Eur. Phys. J. Special Topics 185, 5 (2010)]. 